\title{
Analysis based on CNN for Automated Vehicle Parking Occupancy
}

\author{
Muksimova Shakhnoza \\ Department of Computer Engineering, Gachon University, Gyeonggi-do 461-701 \\ Seongnam, Korea \\ Young Im Cho \\ Department of Computer Engineering, Gachon University, Gyeonggi-do 461-701 \\ Seongnam, Korea \\ E-mail: muksimova1986@gmail.com,yicho@gachon.ac.kr \\ www.gachon.ac.kr
}

\begin{abstract}
The remarkable growth of the automobile industry, along with a lack of urban planning, exacerbated problems such as traffic congestion, air pollution and driving difficulties. The majority of car manufacturers have a preinstalled or aftermarket navigation device in vehicles to make it easier for customers. This helps drivers to travel to their destination with ease. However, a rough estimate of the remaining distance to the destination is given by navigation systems. With the latest advancement in technology, companies are interested to find out whether these advancements can assist in reducing the time spent to search for empty parking space. Locating a vacant parking spot is a big predicament that drivers face because looking for it is a tiring operation. Our investigations have shown that, in contrast with previous methods, for the purpose of classifying given parking spaces as empty or occupied, the current solution is more robust, stable and well-generalized for unseen images taken from entirely separate camera points of view, which has clear signs that it can easily generalize to other parking lots.
\end{abstract}

Keywords: Convolutional neural network, parking, sensors, lightweight network

\section{Introduction}

The value of unoccupied parking space detection systems is growing dramatically, as preventing traffic congestion and the time-consuming task of locating empty parking space is a big challenge for urban center drivers. However, current parking space occupancy monitoring technologies are either costly hardware or not well-generalized for differing images taken from multiple camera views. As a solution, we take advantage of an effective visual identification approach that is made possible by the fact that camera tracking is already present in most parking spaces. The current challenge, on the other hand, is a difficult vision job due to outdoor conditions such as lighting variance, occlusions, distortion of focus, multiple camera views, and adjustments due to the different seasons of the year. In order to address these limitations, we suggest an approach based on the Convolutional Neural Network specially constructed to detect parking space occupancy in a parking lot, provided only the picture of a single parking spot as data.

In addition, the computer vision for traffic applications is an evolving field of study, with major developments over the last few years. For example, intelligent transport management systems, such as those examined in [1], aim to accomplish tasks such as monitoring, detecting and identifying vehicles, as well as higher-level tasks such as recognizing vehicle actions and revealing irregularities. In a vision-based device, any visual node that contains a camera and a transmitter will simultaneously observe several cars, minimizing the cost per parking stand. As it can be used for other activities, such as security, the device base is normally already configured and can be handled by other applications after implementation. However, the literature review [3] has demonstrated that image-based parking reservation tracking policies can be enforced using existing security cameras that are already connected to the main monitoring system. On the opposite, those who support sensor-based approaches, 
such as the authors of [2], contend that video cameras are significantly more costly than sensors and generate a significant quantity of data that cannot be readily disseminated over a wireless network.

In this paper, to resolve the above problems, we are presenting a CNN framework that uses dilated convolution neural networks to show the status of parking space occupancy. The PKLot dataset [4], which is freely available, was used to test the efficiency of our model. Using pictures of various situations and parking lots, we were able to test the generalizability of our strategy. We learned our model on a subset of images and checked it on a totally different one. Several methods already exist, such as the one explored in the analysis mentioned in [5], AlexNet, where this sort of generalization property has been experimentally tested; however, we have shown that our method outperforms them. Most of the related studies contrasted their methodology to Alex Net [6], which showed a high degree of success in this mission. Thus, in conjunction with Alex Net, as well as with other well-known deep learning structures, we tested our method

\section{Related Work}

To address the problem mentioned above, there are currently different schemes, such as those suggested in $[2,7]$. In several studies, the issue with the parking management scheme was resolved by the use of smart devices. Sensor-based solutions include sensors for identification, such as ultrasonic sensors, which are mounted in each parking room. Huang and Wang [9] held that image-based approaches can be divided into two classes: space-driven and car-driven. Space-driven approaches are targeted at finding empty parking spaces rather than vehicles. In the case of images taken from static (such as surveillance) cameras, the technique most commonly used is background subtraction, which implies that the background deviation is statistically immobile over a small time period. Considering that this presumption does not hold outdoor or outdoor scenes, the limitations of this method are instantly apparent. The authors used Gabor filters to train a classifier with images of unoccupied parking spaces under different lighting conditions. In the category of car-driven process, algorithms are learned to identify vehicles that are objects of interest. Due to the distortion of perspective found in most of the parking lot images, as seen in Figure 1, cars positioned at a substantial distance from the camera occupy a small area of the scene, and thus less information can be observed, which greatly decreases the efficiency of the object detection algorithms. The technique presented in [5] is based on a deep Convolutional Neural Network specially developed for smart cameras. They have obtained reasonably high results, and we will equate our results with that. The method, named mAlexNet, is a variant of AlexNet and provides a decentralised approach for visual space occupancy detection.
Very strong results were obtained, but only for the same subset of images.

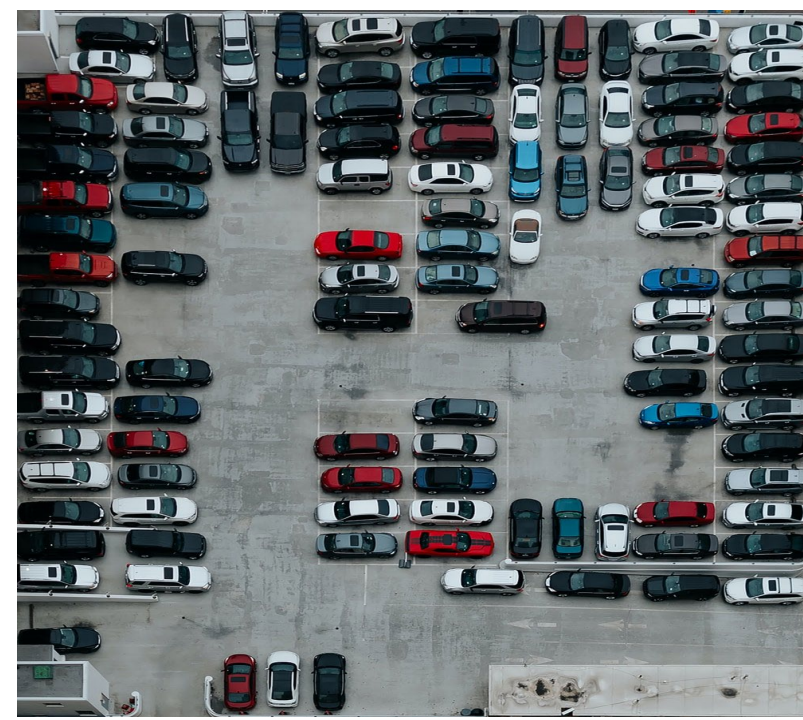

Figure 1. Example for a car parking image from PKLot dataset [4].
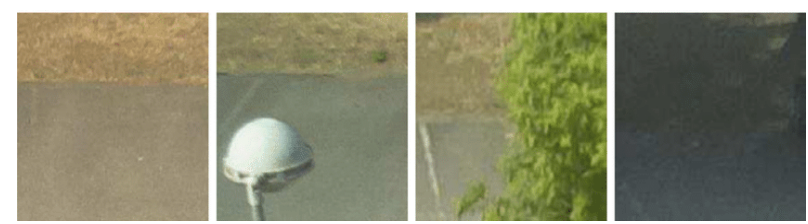

(a)
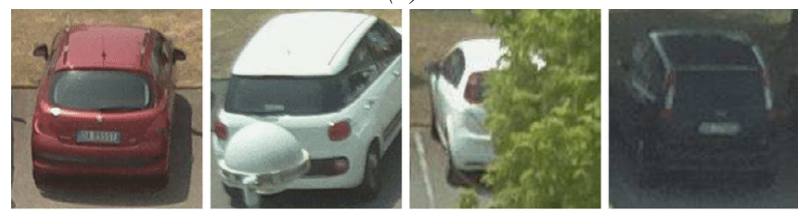

(b)

Figure 2. Segmented images of dataset for occupied and vacant space. 


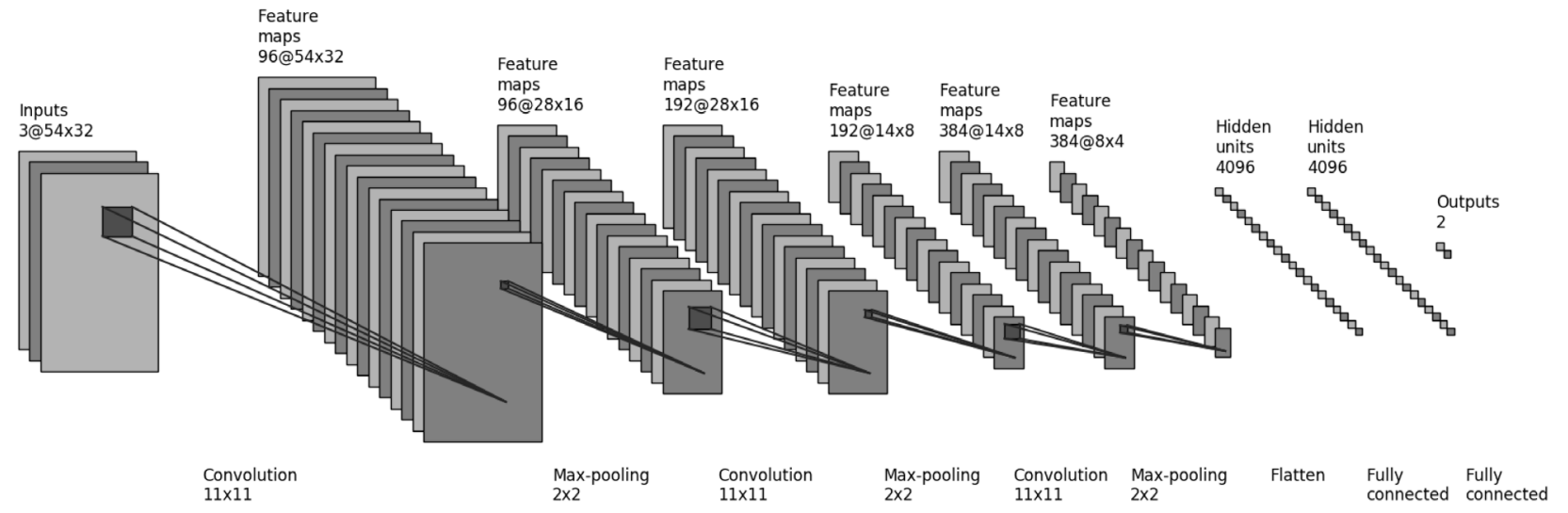

\section{Proposed Approach}

There are three subsets of this PKLot dataset [4] and during this experiment, we only utilized two of them in

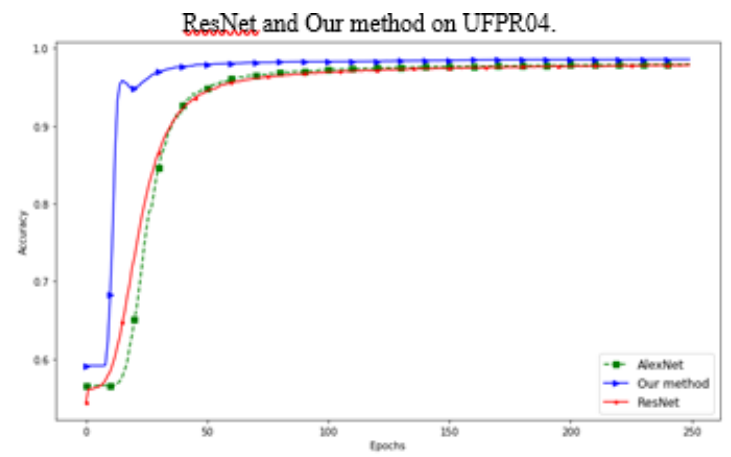

our work. As we mentioned above on this task, AlexNet [6] model was achieved highest performance and so far a number of models were compared to this model in this reason we also compared our work to AlexNet [6] model. Our model is five layers deep and output layer. However, AlexNet [6] model is eight layers deep that five convolutional and three fully connected layers and learning of. parameters are also fairly many than our network. While increasing the number of learnable parameters makes the model bigger and increases training time. Below, in Table 1 and Figure 3 is shown experimental results of the models.

Table 1. Comparison of training and validation scores for AlexNet, ResNet and Our method on UFPR04.

\begin{tabular}{|c|c|c|}
\hline $\begin{array}{l}\text { Name of } \\
\text { Method }\end{array}$ & $\begin{array}{l}\text { Training } \\
\text { Accuracy }\end{array}$ & $\begin{array}{l}\text { Validation } \\
\text { Accuracy }\end{array}$ \\
\hline Our Method & $97.92 \%$ & $97.87 \%$ \\
\hline Alex Net[6] & $96.99 \%$ & $97.91 \%$ \\
\hline Res Net[5] & $96.51 \%$ & $97.80 \%$ \\
\hline
\end{tabular}

Figure 3. Comparison of our model with respect to other models

\section{CONCLUSION}

An efficient solution-Our model, for visual detection of a parking status, was presented which uses Convolutional Neural Networks. Our novel model is provided as a robust design for employees to classify images of parking spaces taken from a camera as occupied or vacant. In this proposed approach, we used three contributions: a little number of layers, small window sizes. We showed by experiments that the current task can be tackled effective by using these contributions. To assess the performance of the model and to be able to compare it with respect to other architectures, we made our experiments on PKLot dataset since prior attends were evaluated on this dataset as well.

\section{Acknowledgements}

This research was supported the National Research Foundation (NRF) - 2018R1D1A1A09084151.

\section{References}

1. Tian, B.; Morris, B.T.; Tang, M.; Liu, Y.; Yao, Y.; Gou, C.; Shen, D.; Tang, S. Hierarchical and Networked Vehicle Surveillance in Its: A Survey. IEEE Trans. Intell. Transp. Syst. 2015, 16, 557-580.

2. Tang, V.W.; Zheng, Y.; Cao, J. An Intelligent Car Park Management System Based on Wireless Sensor Networks. In Proceedings of the 1st International Symposium on Pervasive Computing and Applications, Urumchi, Xinjiang, China, 3-5 August 2006; pp. 65-70.

3. Ichihashi, H.; Notsu, A.; Honda, K.; Katada, T.; Fujiyoshi, M. Vacant Parking Space Detector for Outdoor Parking Lot by Using Surveillance Camera and FCM classifier. In Proceedings of the IEEE International 
Conference on Fuzzy Systems, Jeju Island, Korea, 20-24 August 2009; pp. 127-134.

4. Amato, G.; Carrara, F.; Falchi, F.; Gennaro, C.; Meghini, C.; Vairo, C. Deep Learning for Decentralized Parking Lot Occupancy Detection. Expert Syst. Appl. 2017, 72, 327-334. [CrossRef

5. De Almeida, P.R.; Oliveira, L.S.; Britto, A.S., Jr.; Silva, E.J., Jr.; Koerich, A.L. PKLot-A Robust Dataset for Parking Lot Classification. Expert Syst. Appl. 2015, 42, 4937-4949. [CrossRef].

6. Amato, G.; Carrara, F.; Falchi, F.; Gennaro, C.; Meghini, C.; Vairo, C. Deep Learning for Decentralized Parking Lot Occupancy Detection. Expert Syst. Appl. 2017, 72, 327-334. [CrossRef].

7. Krizhevsky, A.; Sutskever, I.; Hinton, G.E. ImageNet Classification with Deep Convolutional Neural Networks. In Advances in Neural Information Processing Systems 25: 26th Annual Conference on Neural Information Processing Systems 2012; Curran Associates, Inc.: Red Hook, NY, USA, 2012.

8. Ashok, V.G.; Gupta, A.; Shiva, S.; Iyer, H.; Gowda, D.; Srinivas, A. A Novel Parking Solution for Metropolitan Parking Garages. In Proceedings of the 3rd WSEAS International Conference on Urban Planning and Transportation, Sozopol, Bulgaria, 22-24 July 2010; pp. 153-159.

9. Huang, C.C.; Wang, S.J. A Hierarchical Bayesian Generation Framework for Vacant Parking Space Detection. IEEE Trans. Circuits Syst. Video Technol. 2010, 20, 1770-1785. [CrossRef] 